\title{
An efficient thermal diode with ballistic spacer
}

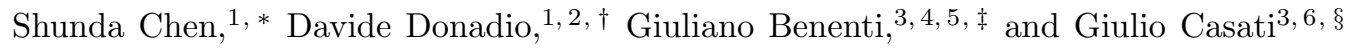 \\ ${ }^{1}$ Department of Chemistry, University of California Davis, One Shields Ave. Davis, CA 95616, USA \\ ${ }^{2}$ Ikerbasque, Basque Foundation for Science, E-48011 Bilbao, Spain \\ ${ }^{3}$ Center for Nonlinear and Complex Systems, Dipartimento di Scienza e Alta Tecnologia, \\ Università degli Studi dell'Insubria, via Valleggio 11, 22100 Como, Italy \\ ${ }^{4}$ Istituto Nazionale di Fisica Nucleare, Sezione di Milano, via Celoria 16, 20133 Milano, Italy \\ ${ }^{5}$ NEST, Istituto Nanoscienze-CNR, I-56126 Pisa, Italy \\ ${ }^{6}$ International Institute of Physics, Federal University of Rio Grande do Norte, \\ Campus Universitário - Lagoa Nova, CP. 1613, Natal, Rio Grande Do Norte 59078-970, Brazil
}

(Dated: April 3, 2022)

\begin{abstract}
Thermal rectification is of importance not only for fundamental physics, but also for potential applications in thermal manipulations and thermal management. However, thermal rectification effect usually decays rapidly with system size. Here, we show that a mass-graded system, with two diffusive leads separated by a ballistic spacer, can exhibit large thermal rectification effect, with the rectification factor independent of system size. The underlying mechanism is explained in terms of the effective size-independent thermal gradient and the match/mismatch of the phonon bands. We also show the robustness of the thermal diode upon variation of the model's parameters. Our finding suggests a promising way for designing realistic efficient thermal diodes.
\end{abstract}

PACS numbers: 05.70.Ln; 05.60.-k; 44.10.+i

Introduction. Heat is a ubiquitous form of energy, of which we have limited control. Waste heat limits the performance of the smallest chips present in electronic devices, such as laptop computers and cellular phones. Energy supply and cooling is also a challenge for the large data centers and supercomputers. Therefore, efficiently harnessing thermal energy would have an enormous societal impact. Effective control of heat currents requires the development of a new class of nanoscale thermal devices, namely thermal rectifier and amplifiers, analogous to electronic diodes and transistors.

Nonlinear dynamics indicates possible pathways toward thermal diodes and transistors [1 5 . However, in spite of pioneering experimental investigations of thermal rectification of phonon transport [6]12, a satisfactory real-life implementation of these concepts has not been achieved yet [13.

The rectification factor can be defined as

$$
f_{r}=\frac{\left(J_{+}-J_{-}\right)}{J_{-}} \times 100 \%,
$$

where $J_{+}$and $J_{-}$represent, respectively, the larger heat flow and the smaller heat flow, obtained by inverting the temperature bias applied to the system. While numerical simulations of theoretical models of nonlinear systems predict rectification factors of the order of $10000 \%$, experimental phononic devices so far are limited to $f_{r} \approx 70 \%$ 6-13.

A general and yet unsolved problem of thermal rectifiers is that rectification rapidly decays to zero as the size increases. This effect is at first sight unavoidable since, for a given temperature bias, the temperature gradient decreases as the system size increases. Consequently, the linear response regime where rectification vanishes should be approached. From a practical viewpoint, it would be highly desirable to overcome this problem, since it is difficult to apply large temperature biases on small sizes. A first proposal [14, 15] was based on mass-graded system with long-range interactions, where new channels (interactions) among different sites with different masses were created, due to the introduction of long-range interactions. The long range interactions (new channels) in mass-graded system connect distant particles with very different masses, leading to the increase of asymmetry, which favor the asymmetric flow, i.e. rectification, and avoid the usual decay of rectification with system size. The idea of introducing new channels is inspiring, and further motivates us to look for experimentally feasible ways to have large thermal rectification effect with the rectification factor independent of the system size.

In this Letter, we consider a one-dimensional, segmented mass-graded system, with the particles in the outer parts of the system (hereafter referred to as left and right lead) exposed to nonlinear on-site potentials, and separated by a ballistic channel, where the interaction between the particles is harmonic. We show that this model exhibits large rectification. Even more importantly, thanks to the ballistic spacer, the size dependence of the rectification factor is removed. That is, $f_{r}$ does not decay with the system size. The rectification is explained in terms of the match/mismatch (depending on the direction of the applied thermal bias) of the phonon bands for the two leads and for the ballistic channel. We also show that our results are quite robust upon variation of the system parameters and that our model is flexible. Indeed the main ingredient for rectification is the different temperature dependence of the phonon bands in the two leads, and this effects can be obtained in several 


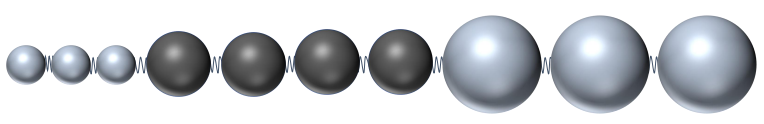

$\phi^{4}$ lattice $\begin{gathered}\text { Harmonic lattice } \\ \text { (Ballistic Channel) }\end{gathered} \quad \phi^{4}$ lattice

FIG. 1: Schematic drawing of our model. It consists of three parts, one central ballistic channel with $N_{C}$ particles of mass $m_{C}$, and two leads $\left(\phi^{4}\right.$ lattices $)$, with $N_{L}\left(N_{R}\right)$ particles of mass $m_{L}\left(m_{R}\right)$ and on-site potential strength $\gamma_{L}\left(\gamma_{R}\right)$ in the left (right) lead.

ways. For instance, with different on-site potentials in the left and right leads rather than with a mass-graded system. Finally, we show that large rectification for large system sizes can still be obtained if the spacer is subballistic rather than ideally ballistic. The stability of the effect upon nonlinear interactions in the spacer is relevant in view of possible experimental implementations. Prospects in this regard are also discussed.

The model. The system we will discuss here (schematically drawn in Fig. 1) is a one-dimensional chain of $N_{\text {tot }}$ oscillators described by the Hamiltonian

$$
H=\sum_{i=1}^{N_{\text {tot }}}\left(\frac{p_{i}^{2}}{2 m_{i}}+\frac{\gamma_{i} q_{i}^{4}}{4}\right)+\frac{1}{2} \sum_{i=1}^{N_{\text {tot }}-1}\left(q_{i+1}-q_{i}\right)^{2},
$$

where $q_{i}$ is the displacement from the equilibrium position of the $i$-th particle with mass $m_{i}$ and momentum $p_{i}$, and $\gamma_{i}$ measures the strength of the on-site quartic potential. The systems consists of $N_{L}\left(N_{R}\right)$ particles with mass $m_{L}\left(m_{R}\right)$ and strength of the on-site potential $\gamma_{L}$ $\left(\gamma_{R}\right)$ in the left (right) lead. These two anharmonic leads ( $\phi^{4}$ lattices) are connected by a ballistic channel, that is, by a purely harmonic central chain of $N_{C}$ particles with mass $m_{C}$ and zero on-site potential, $\gamma_{C}=0$. The total system size is $N_{\text {tot }}=N_{L}+N_{C}+N_{R}$.

In our non-equilibrium simulations, two Langevin heat baths (with dissipation coefficient of 1) 16 at different temperatures $T_{-}$and $T_{+}$are attached to the two ends of the leads [leftmost (rightmost) particle of left (right) lead]; we take $T_{-}=T\left(1-\frac{\Delta T}{T}\right)$ and $T_{+}=T\left(1+\frac{\Delta T}{T}\right)$. We investigate the thermal transport properties of our model, focusing in particular on the rectification factor $f_{r}$, defined in Eq. (1).

In our simulations, each system is evolved for a long enough time $\left(>10^{8}\right)$ to ensure that it has reached the stationary state. After that the heat current is measured. The simulation time for averaging heat flux is larger than $10^{10}$. More specifically, we verified the average heat flow through each particle is the same (within $0.01 \%$ ). Free boundary conditions and velocity-Verlet algorithm (with time step 0.01) are used. We have checked simulations using fixed boundary conditions and simulations using Runge-Kutta algorithm of seventh to eighth order, and

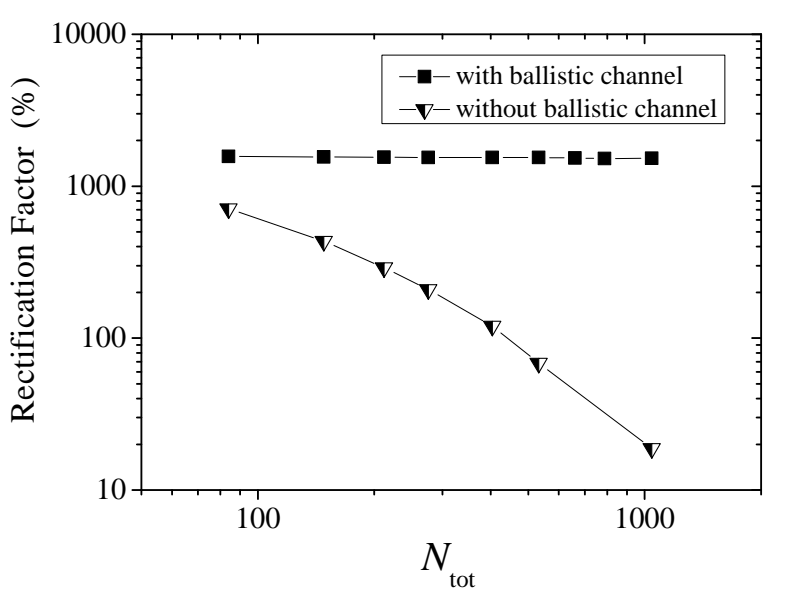

FIG. 2: Thermal rectification factor $f_{r}$ versus system size $N_{\text {tot }}$ for chains with or without ballistic channel (squares are for $\phi^{4}$ harmonic- $\phi^{4}$ model, i.e. with ballistic channel, and triangles are for $\phi^{4}-\phi^{4}-\phi^{4}$ model, i.e. without ballistic channel). Here, $T_{+}=9.5, T_{-}=0.5, N_{L}=N_{R}=10, m_{L}=1, m_{C}=4.5$, $m_{R}=10, \gamma_{L}=\gamma_{R}=1$, and $\gamma_{C}=0\left(\gamma_{C}=1\right)$ for the model with (without) ballistic channel.

obtained consistent results. As commonly adopted in the literature, we use dimensionless units.

Size-independent rectification. We start our numerical studies by highlighting the role played by the ballistic channel. For that purpose, we compare in Fig. 2 the sizedependence of the rectification factor $f_{r}$ for model (2), when the intermediate channel is either diffusive $\left(\gamma_{C}=\right.$ $\left.\gamma_{L}=\gamma_{R}\right)$ or ballistic $\left(\gamma_{C}=0\right)$. While in the former case $\left(\phi^{4}-\phi^{4}-\phi^{4}\right.$ model) the rectification factor rapidly decays with the system size, in the latter case $\left(\phi^{4}\right.$-harmonic- $\phi^{4}$ model) the rectification factor is size-independent. Note that we found the larger heat flow $J_{+}$when the higher temperature $T_{+}$was on the side of the heavier masses as in previous work [6, 15.

In Fig. 3. we compute the temperature profile inside the system, that is, the local temperature at site $i$ $\left(i=1, \ldots, N_{\text {tot }}\right)$ defined as $T_{i}=m_{i}\left\langle\dot{q}_{i}^{2}\right\rangle$, where $\langle$.$\rangle stands$ for temporal average under steady-state conditions. The different behavior of the rectification factor as a function of the system size with and without an intermediate ballistic channel is accompanied by a striking difference in the local temperature profile. In both cases, the temperature profile exhibits a marked asymmetry between forward (left-right) and backward (right-left) temperature bias, as needed to have large rectification factors. For the model with a ballistic channel, the temperature profile is flat inside the central, harmonic region, with a value of the temperature which is size-independent. This implies that also the temperature gradients in the two leads are size independent. On the other hand, for the model without ballistic channel the temperature profile is such that the temperature gradients reduce with the sys- 

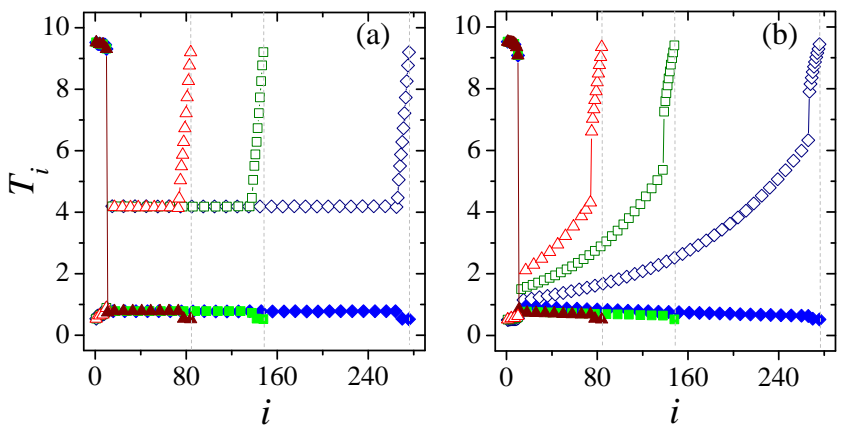

FIG. 3: (Color online) (a) Temperature profiles, both for forward (left-right, full symbols) and backward (right-left, empty symbols) temperature bias, for the model with a ballistic channel ( $\phi^{4}$-harmonic- $\phi^{4}$ model $)$ and (b) for the model without a ballistic channel $\left(\phi^{4}-\phi^{4}-\phi^{4}\right.$ model). In both cases $N_{\text {tot }}=84$ (triangles), 148 (squares), and 276 (diamonds), respectively. Here $T_{+}=9.5, T_{-}=0.5, m_{L}=1, m_{R}=10$, $m_{C}=4.5, N_{L}=N_{R}=10, \gamma_{L}=\gamma_{R}=1$, and $\gamma_{C}=0$ (panel (a)) or $\gamma_{C}=1$ (panel (b)).

tems size. As a consequence, the linear response regime is approached and the rectification factor decreases with the system size.

Rectification mechanism. The numerically observed thermal rectification can be explained in terms of the match/mismatch of the phonon bands for the two leads, when the higher temperature is on the side of the heavier/lighter masses. We compute the vibrational power spectrum as the Fourier transform of the velocity-velocity autocorrelation function of a particle:

$$
P(\omega)=\int\langle v(t) v(0)\rangle e^{-i \omega t} d t
$$

where the simulations are for a closed $\phi^{4}$ system, with particle mass $m$, periodic boundary conditions, and at thermal equilibrium. An example of power spectrum is shown in Fig. 4(a). The numerical results are in good agreement with those obtained from the effective phonon approach [17, 18. For a $\phi^{4}$ lattice, this approach predicts an effective (i.e., renormalized by the nonlinearity) phonon spectrum in the band $\sqrt{1.23 T^{2 / 3} / m} \leq$ $\omega \leq \sqrt{\left(4+1.23 T^{2 / 3}\right) / m}$ 18. This estimated phonon band is shown as a function of temperature in Fig. 4(b). The temperature dependence is stronger for light masses, while for heavy masses the band is almost flat, since the nonlinearity in this case is weak. Therefore, as shown in Fig. 4(c)-(d) we have larger overlapping of the power spectrum for the two leads (and consequently higher thermal conductivity) when the higher temperature is applied to the heavier lead. We note that a similar match/mismatch of the vibrational power spectrum is obtained from the nonequilibrium simulations of the entire $\phi^{4}$-harmonic- $\phi^{4}$ model exposed to thermal baths, comparing the power spectrum for two particles, one in the
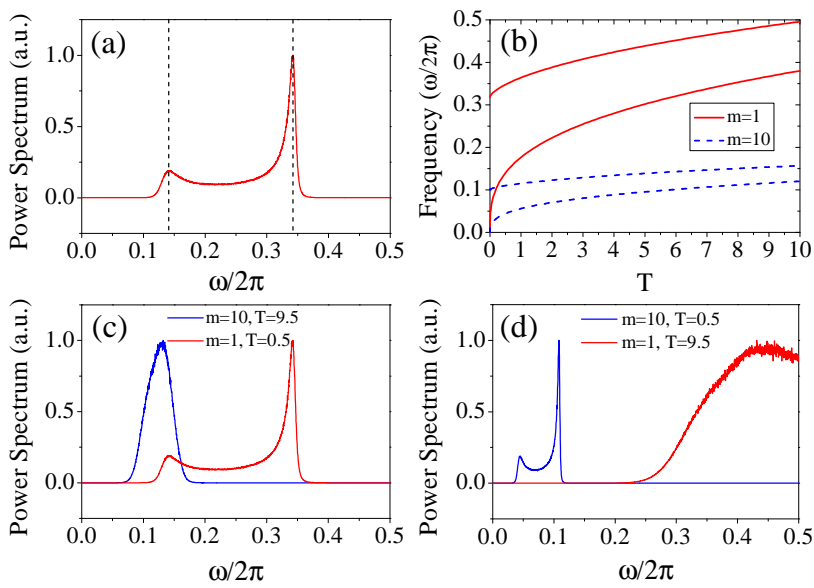

FIG. 4: (Color online) Vibrational power spectrum for the $\phi^{4}$ lattice, calculated at thermal equilibrium at temperature T. (a) $m=1, T=0.5$; the dashed lines correspond to the boundaries of the analytically estimated phonon band. (b) Analytically estimated phonon band as a function of temperature, for $m=1$ (red solid lines) and $m=10$ (blue dashed lines). (c) Power spectrum for $m=10, T=9.5$ (blue line) and for $m=1, T=0.5$ (red line). (d) Power spectrum for $m=10, T=0.5$ (blue line) and for $m=1, T=9.5$ (red line). In all cases the nonlinearity strength $\gamma=1$.

lighter lead and the other in the heavier lead (data not shown here).

We also point out that the rectification factor is optimized when the mass of the ballistic channel is chosen in such a way that its phonon band has a significant overlap with the phonon bands of both leads (for the thermal bias direction corresponding to the larger thermal current $\left.J_{+}\right)$. This happens for a value of $m_{C}$ intermediate between $m_{L}$ and $m_{R}$. More precisely, for the masses and temperatures chosen in our simulations, we found that the optimal value for rectification is $m_{C} \approx 4.5$ (see Fig. 5 p.

Robustness of rectification. It is important to study the robustness of the rectification effect upon variations of the system's parameters. In Fig. 5 (a) we change the mass $m_{L}$ of the lighter particles. We can see that, while the rectification factor is huge for large mass difference, $m_{L} / m_{R} \ll 1$, a significant effect still remains for moderate mass asymmetry. In Fig. 5(b) we show that a large rectification is observed in a broad range of values for $m_{C}$ around the optimal value $m_{C} \approx 4.5$, including the cases when $m_{C}$ approaches either $m_{L}$ or $m_{R}$.

In Fig. 6(a) we show that, even in the absence of mass gradient $\left(m_{L}=m_{R}=m_{C}\right)$ a moderately high rectification, $f_{r} \approx 200 \%$, is possible when the needed asymmetry in the system is provided by the on-site potential, that is, $\gamma_{L} / \gamma_{R} \gg 1$. If we add large asymmetry for the on-site potential, on top of a mass-graded system, the rectification can become very large. For instance, in Fig. 6(b) we obtain $f_{r} \approx 3700 \%$ for $\gamma_{L}=10 \gamma_{R}$ and $m_{R}=10 m_{L}$.

We finally investigate the robustness of the rectifica- 

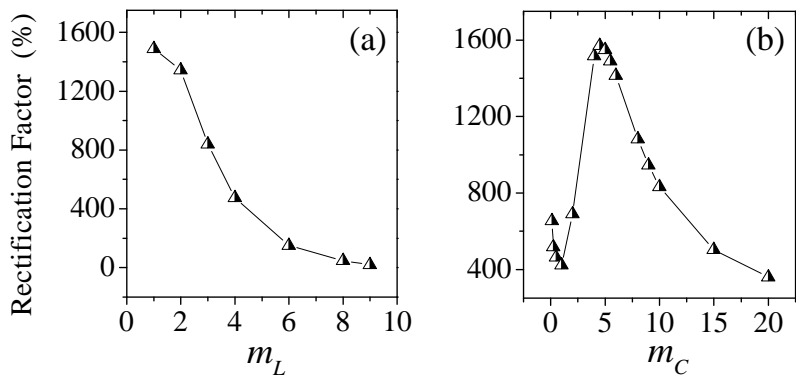

FIG. 5: Dependence of the rectification factor on: (a) mass gradient $\left(m_{R}=10, m_{C}=\left(m_{L}+m_{R}\right) / 2, T_{+}=9.5, T_{-}=0.5\right)$ and (b) mass of the particles in the ballistic channel $\left(m_{L}=1\right.$, $\left.m_{R}=10, T_{+}=9.5, T_{-}=0.5\right)$. In both panels, $N_{L}=N_{R}=$ $10, N_{C}=64$, total system size $N_{\text {tot }}=84, \gamma_{L}=\gamma_{R}=1$.
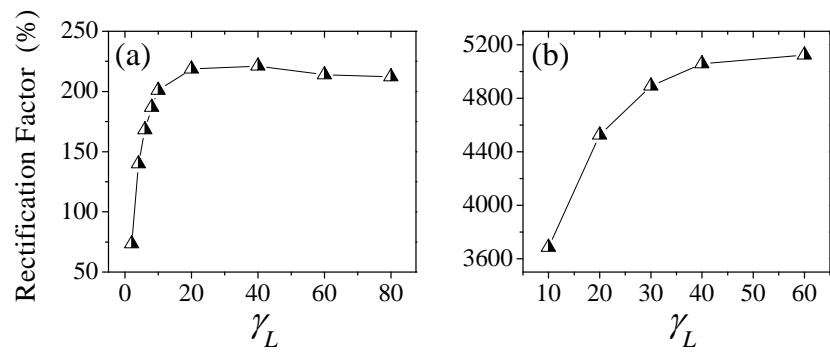

FIG. 6: (a) Dependence of the rectification factor on the strength $\gamma_{L}$ of the on-site potential in the left lead for (a) the equal-mass system $\left(m_{L}=m_{R}=m_{C}=1\right)$ and (b) the mass-graded system $\left(m_{L}=1, m_{R}=10, m_{C}=5.5\right)$. In both panels, $N_{L}=N_{R}=10, N_{C}=64$, total system size $N_{\text {tot }}=84$, $T_{+}=9.5, T_{-}=0.5, \gamma_{R}=1$.

tion when the central channel is not ballistic as in the ideal case, but anharmonicity is present, modeled by the Fermi-Pasta-Ulam (FPU) Hamiltonian FPU- $\beta$. That is, we add to the Hamiltonian for the $N_{C}$ particles in the spacer the anharmonic term $\sum_{i} \frac{\beta}{4}\left(q_{i}-q_{i-1}\right)^{4}$. As a consequence the channel is sub-ballistic [16, 19] and, as shown in Fig. 7(a), the rectification factor decays with the system size. However, the decay is much slower than for a diffusive, $\phi^{4}$ central channel, also shown for a comparison in the same figure. We also show in Fig. 7(b) that, for a given system size, rectification remains large up to some value of the nonlinearity parameter $\beta$. For instance, we can see that for $N_{\text {tot }}=84$ we have $f_{r} \gtrsim 1000 \%$ for $\beta \lesssim 0.5$.

Conclusions. To summarize, we have discussed a model of a thermal diode, consisting of two nonlinear systems with different masses and/or different on-site potentials, coupled by a ballistic thermal conductor. The ballistic channel removes the size dependence of the rectification factor, thus opening new possibilities of achieving high rectification factors in experimental devices.

We have also checked that the same main features of our system (high and size-independent rectification) can be obtained in another model, with a ballistic chan-
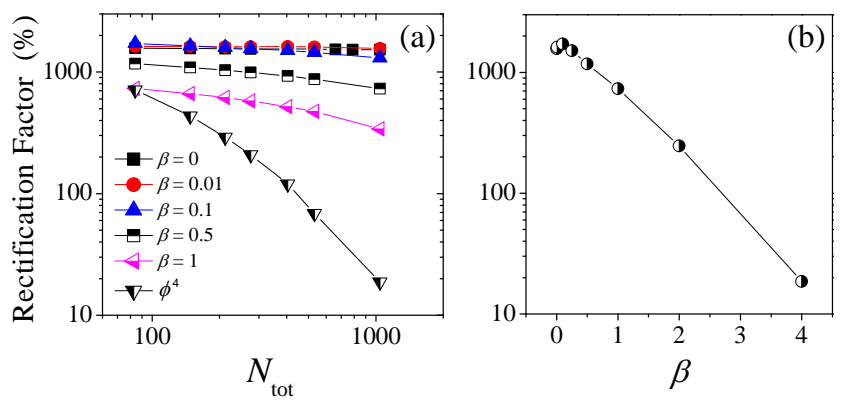

FIG. 7: (Color online) Effect of the anharmonicity (FPU- $\beta$ model) of the central channel on the rectification. (a) Rectification factor versus system size for different values of $\beta$ ( $\beta=0$ corresponds to the ballistic, harmonic channel); the case of a diffusive, $\phi^{4}$ central channel is also shown for a comparison. (b) Rectification factor versus anharmonicity strength for size of the central channel $N_{C}=64$. In both panels, $N_{L}=N_{R}=10, m_{L}=1, m_{R}=10, m_{C}=4.5, T_{+}=9.5$, $T_{-}=0.5, \gamma_{L}=\gamma_{R}=1$.

nel (harmonic lattice) connected to a single mass-graded nonlinear $\left(\phi^{4}\right)$ lead, consisting of $N_{R}$ particles with masses increasing from $m_{\min }$ to $m_{\max }$. This shows the conceptual simplicity and flexibility of models based on mass gradient plus a ballistic channel for thermal transport.

We expect that our results might be exploited to design a realistic thermal diode. In a recent work [12, it was demonstrated experimentally thermal rectification in various asymmetric monolayer graphene nanostructures. A thermal rectification factor as large as $26 \%$ is reported in a defect-engineered asymmetric monolayer graphene with nanopores on one side. However, as demonstrated by molecular dynamics simulations in the same work, the thermal rectification factor would decay rapidly with system size. It is thus interesting if one could have possible experimental implementations considering the ballistic spacer model we discussed here, to prevent the fast decay of rectification with system size. Since graphene has very high thermal conductivity 20 23 , one could consider graphene as quasi-ballistic spacer, and apply asymmetry on its two ends, where one could consider several asymmetric ingredients, such as defects, geometry, mass-loading, chemical functionalization, substrate couplings, mechanical strains, etc. One could also consider connecting graphene to heavier leads, such as Molybdenum disulfide $\left(\mathrm{MoS}_{2}\right)$ 24] or Tungsten diselenide $\left(\mathrm{WSe}_{2}\right)$ 25, creating van der Waals heterostructures [26, 27] with tunable interlayer coupling strength (anharmonicity) through stress or mechanical strain. Besides graphene, one could also consider other high thermal conductivity materials, such as carbon nanotube 2831 or carbyne [32, 33], as quasi-ballistic spacer, and create asymmetry on top of them to build efficient thermal 
diodes.

Acknowledgments: We acknowledge the support by the CINECA project Nanostructures for Heat Management and Thermoelectric Energy Conversion.

* Electronic address: shdchen@ucdavis.edu

† Electronic address: ddonadio@ucdavis.edu

¥ Electronic address: giuliano.benenti@uninsubria.it

$\S$ Electronic address: giulio.casati@uninsubria.it

[1] M. Terraneo, M. Peyrard M., and G. Casati, Phys. Rev. Lett., 88, 094302 (2002).

[2] B. Li, L. Wang, and G. Casati, Phys. Rev. Lett. 93, 184301 (2004).

[3] B. Li, L. Wang, and G. Casati, Appl. Phys. Lett. 88, 143501 (2006).

[4] N. Li, J. Ren, L. Wang, G. Zhang, P. Hänggi, and B. Li, Rev. Mod. Phys. 84, 1045 (2012).

[5] G. Benenti, G. Casati, C. Mejía-Monasterio, and M. Peyrard, From thermal rectifiers to thermoelectric devices, in Thermal transport in low dimensions, S. Lepri (Ed.), Lecture Notes in Physics 921 (Springer, 2016).

[6] C. W. Chang, D. Okawa, A. Majumdar, and A. Zettl, Science 314, 1121 (2006)

[7] W. Kobayashi, Y. Teraoka, and I. Terasaki, Appl. Phys. Lett. 95, 171905 (2009).

[8] D. Sawaki, W. Kobayashi, Y. Morimoto, and I. Terasaki, Appl. Phys. Lett. 98, 081915 (2011).

[9] W. Kobayashi, D. Sawaki, T. Omura, T. Katsufuji, Y. Moritomo, and I. Terasaki, Appl. Phys. Express 5, 027302 (2012).

[10] M. Schmotz, J. Maier, E. Scheer, and P. Leiderer, New J. Phys. 13, 113027 (2011).

[11] H. Tian, D. Xie, Y. Yang, T. L. Ren, G. Zhang, Y. F. Wang, C. J. Zhou, P. G. Peng, L. G. Wang, and L. T. Liu, Sci. Rep. 2, 523 (2012).

[12] H. Wang, S. Hu, K. Takahashi, X. Zhang, H. Takamatsu, and J. Chen, Nature Comm. 8, 15843 (2017).

[13] We do not discuss here the thermal rectification of photonic or electronic currents; in particular, large rectification of electronic heat current in a hybrid device com- bining normal metals tunnel-coupled to superconductors, was reported in M. J. Martinez-Pérez, A. Fornieri, and F. Giazotto, Nat. Nanotechnol. 10, 303 (2015).

[14] E. Pereira and R. R. Ávila, Phys. Rev. E 88, 032139 (2013).

[15] S. Chen, E. Pereira, and G. Casati, EPL 111, 30004 (2015).

[16] A. Dhar, Adv. Phys. 57, 457 (2008).

[17] D. Boyanovsky, C. Destri, and H. J. de Vega, Phys. Rev. D 69, 045003 (2004).

[18] N. Li and B. Li, Phys. Rev. E 87, 042125 (2013).

[19] S. Lepri, R. Livi, and A. Politi, Phys. Rep. 377, 1 (2003).

[20] A. A. Balandin, S. Ghosh, W. Bao, I. Calizo, D. Teweldebrhan, F. Miao, and C. N. Lau, Nano Lett. 8, 902 (2008).

[21] A. A. Balandin, Nat. Mater. 10, 569 (2011).

[22] L. F. C. Pereira and D. Donadio, Phys. Rev. B 87, 125424 (2013).

[23] X. Xu, L. F. C. Pereira, Y. Wang, J. Wu, K. Zhang, X. Zhao, S. Bae, C. T. Bui, R. Xie, J. T. L. Thong, B. H. Hong, K. P. Loh, D. Donadio, B. Li, and B. Özyilmaz, Nature Comm. 5, 3689 (2014).

[24] K. F. Mak, C. Lee, J. Hone, J. Shan, and T. F. Heinz, Phys. Rev. Lett. 105, 136805 (2010).

[25] C. Chiritescu, D. G. Cahill, N. Nguyen, D. Johnson, A. Bodapati, P. Keblinski, and P. Zschack, Science 315, 351 (2007).

[26] A. K. Geim and I. V. Grigorieva, Nature 499, 419 (2013).

[27] K. S. Novoselov, A. Mishchenko, A. Carvalho, and A. H. C. Neto, Science 353, 461 (2016).

[28] P. Kim, L. Shi, A. Majumdar, and P. L. McEuen, Phys. Rev. Lett. 87, 215502 (2001).

[29] E. Pop, D. Mann, Q. Wang, K. Goodson, and H. Dai, Nano Lett. 6, 96 (2006).

[30] D. Donadio and G. Galli, Phys. Rev. Lett. 99, 255502 (2007).

[31] L. F. C. Pereira and D. Donadio, Phys. Rev. B 87, 125424 (2013).

[32] M. Wang and S. Lin, Sci. Rep. 5, 18122 (2015).

[33] L. Shi, P. Rohringer, K. Suenaga, Y. Niimi, J. Kotakoski, J. C. Meyer, H. Peterlik, M. Wanko, S. Cahangirov, A. Rubio, Z. J. Lapin, L. Novotny, P. Ayala, and T. Pichler, Nat. Mater. 15, 634 (2016). 